\title{
Growth and Growth Factors
}

\author{
Evelien F Gevers ${ }^{a, b}$, Peter C Hindmarsh ${ }^{a}$ and Mehul T Dattania \\ aDevelopmental Endocrine Research Group, Clinical and Molecular Genetics Unit, Institute for Child Heath, \\ London, UK

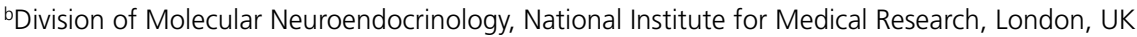

\begin{abstract}
The past year was once again a fruitful year regarding research into growth and growth factors. Exciting new developments include new mutations causing Noonan syndrome, all of which increase RAS signalling, as well as studies succeeding in altering imprinting of IGF-2 in tumor cells and studies shedding light on regulation of a network of imprinted genes that is involved in embryonic growth. Long-range control of gene transcription was studied in detail for the growth hormone (GH) and SHOX genes and the importance of such regulation was suggested by the presence of deletions downstream of the SHOX-coding region in patients with Leri-Weill dyschondrosteosis. We have learnt more about GH signalling; the importance of negative regulation of cytokine signalling by SOCS2 and JAK2, and the intricate relations between Stats and hepatic nuclear factors that are important for gene transcription in response to $\mathrm{GH}$. Several new animal models were born, including mice overexpressing IGFBP-1 or IGF-1 in the liver, and nude mice transduced with adenoviral vectors containing luciferase reporter genes for Stat5. Clinical trials have suggested efficacy of GH treatment in patients with SHOX mutations, but contradicting results were reported regarding the response to $\mathrm{GH}$ treatment in patients carrying the exon-3-deleted variant of the $\mathrm{GH}$ receptor. Last, but not least, important data from cohort studies were published regarding the long-term follow-up of children with IUGR and SGA.
\end{abstract}

\section{Mechanism of the year Noonan syndrome}

\section{Germline KRAS mutations cause Noonan syndrome}

Schubbert S, Zenker M, Rowe SL, Boll S, Klein C, Bollag G, van der Burgt I, Musante L, Kalscheuer V, Wehner LE, Nguyen H, West B, Zhang KYJ, Sistermans E, Rauch A, Niemeyer CM, Shannon K, Kratz CP Department of Pediatrics, University of California, San Francisco, Calif., USA christian.kratz@uniklinik-freiburg.de Nat Genet 2006;38:331-336

Background: Noonan syndrome, a disorder characterized by short stature, facial dysmorphism and cardiac defects, has previously been linked to gain-of-function mutations in PTPN11, a gene which encodes SHP-2, a non-receptor protein tyrosine phosphatase. However, mutations in this gene account for $\sim 50 \%$ of cases of Noonan syndrome. The aim of this study was to establish whether mutations in $K R A S$, another component of the RAS-signalling pathway, could contribute to the etiology of Noonan syndrome.

Methods: Mutational screening of 174 individuals with Noonan syndrome and 12 patients with cardiofacio-cutaneous (CFC) syndrome. Mutations identified were then studied further using intrinsic and GAP-stimulated GTP hydrolysis assays, retroviral transduction and hematopoietic progenitor assays and Ras-GTP assays.

Results: Three different heterozygous mutations (T58I, V14I and D153V) were identified in 5 patients with Noonan syndrome; a P34R mutation was identified in a patient with CFC syndrome. The functional consequences of the V14I and T58I were studied, and the proteins showed defective intrinsic GTPase activity and impaired responsiveness to GAPs. Additionally, the two mutations rendered primary hematopoietic progenitors hypersensitive to growth factors and deregulated signal transduction in a cell-lineage-specific manner.

Conclusion: These studies and those previously published showing the role of SHP-2 mutations in Noonan syndrome suggest that hyperactive Ras is a critical biochemical lesion in Noonan syndrome. 


\section{Gain-of-function SOS1 mutations cause a distinctive form of Noonan syndrome}

Tartaglia M, Pennacchio LA, Zhao C, Yadav KK, Fodale V, Sarkozy A, Pandit B, Oishi K, Martinelli S, Shackwitz W, Ustaszewska A, Martin J, Bristow J, Carta C, Lepri F, Neri C, Vasta I, Gibson K, Curry CJ, Siguero JPL, Digilio MC, Zampino G, Dallapiccola B, Bar-Sagi D, Gelb BD

Dipartimento di Biologia Cellulare e Neuroscienze, Istituto Superiore di Sanita, Rome, Italy

bruce.gelb@mssm.edu

Nat Genet 2007;39:75-79

Background: The ligand-dependent conversion of RAS-GDP to RAS-GTP is a critical step in the activation of the RAS-MAPK pathway. This reaction is catalyzed by the RAS-specific guanine nucleotide exchange factor (GEF) Son of Sevenless (SOS). SOS1, one of two human SOS proteins, is basally autoinhibited owing to complex regulatory intra- and intermolecular interactions. This study established whether SOS1 mutations contribute to the etiology of Noonan syndrome.

Methods: 129 patients with Noonan syndrome in whom no mutation had been identified in the established genes associated with the syndrome were screened for mutations in SOS1.

Results: Mutations were identified in 22 of 129 individuals. The mutations led to aminoacid substitutions at residues implicated in the maintenance of SOS1 in its autoinhibited form. Functional studies performed on two of the mutants revealed enhanced RAS and ERK activation. Although the phenotype associated with SOS1 mutations is within the Noonan spectrum, the patients manifest generally normal development and linear growth. However, there is a high incidence of pulmonary valve anomalies and ectodermal abnormalities such as facial keratosis pillaris and curly hair.

Conclusion: Gain-of-function mutations in SOS1 are associated with upregulation of the RAS pathway and consequent Noonan syndrome.

About half of the patients with Noonan-syndrome carry mutations in PTPN11, a protein tyrosine phosphatase that functions in the RAS pathway. The Ras-signalling system centers on Ras proteins like H-RAS, K-RAS and N-RAS (fig. 1). RAS proteins are intermediates in a signal transduction pathway that initiates with phosphorylation of a tyrosine kinase receptor in response to an extracellular signal. The phosphorylated tyrosine kinase receptor forms protein complexes with GEFs (guanine nucleotide exchange factors) and GAPs (GTPase-activating proteins) which respectively activate and inactivate RAS. Activated RAS (RAS-GTP) stimulates the mitogen-activated protein kinase (MAPK)signalling pathway which is involved in cell proliferation. PTPN11 mutations found in Noonan syndrome result in prolonged signal flux through the RAS-MAPK pathway. Several research groups argued that mutations of other components of the RAS pathway may also result in Noonan syndrome or cardiofacio-cutaneous (CFC) syndrome of which the features overlap with those of Noonan syndrome. Indeed, the first paper, and a similar paper by Carta et al. [1] shows that mutations in K-RAS are found in Noonan syndrome and CFC syndrome. The mutations result in an increased active RAS-GTP state and a decreased sensitivity for GAPs, thus resulting in increased RAS-MAPK signalling. Tartaglia et al. found SOS-1 mutations in patients with Noonan syndrome. Son-of-Sevenless (SOS) proteins are GEFs that catalyze activation of RAS (RAS-GTP) and so activate MAPK signalling. The SOS-1 mutations found by Tartaglia, and also by Roberts et al. [2], are gain-of-function mutations resulting in increased RAS activation and MAPK signalling. Mutations in both $K-R A S$ and SOS-1 together with PTPN11 in total account for up to $60 \%$ of patients with Noonan syndrome. Mutations in other components of this pathway will probably account for the remainder of cases. Linear growth of patients with SOS1 mutations is often normal linear but they have more pulmonary valve abnormalities. It will be of clinical importance to further evaluate genotype-phenotype correlations in order to give patients with Noonan syndrome and related syndromes optimal care. Activating PTPN11 mutations activate RAS-MAPK signalling but inhibit GH signalling by interacting with the GH receptor and result in mild GH resistance. Mutations in K-RAS and SOS-1 are less likely to affect JAK-Stat signalling directly [3]. 


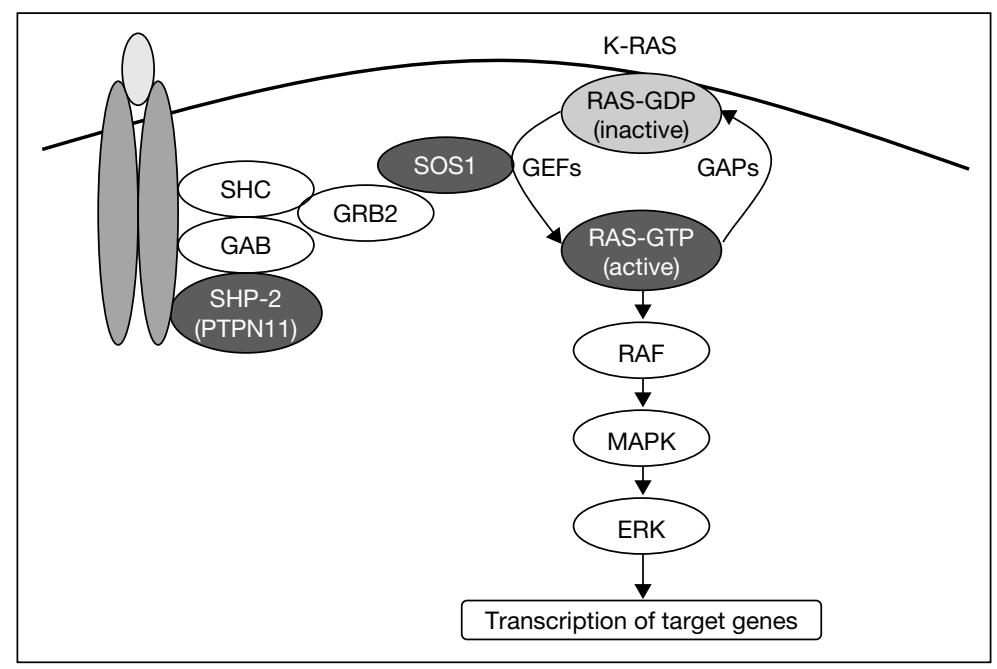

Fig. 1. Simplified schematic diagram showing the RAS-MAPK signal transduction pathway. Mutations resulting in Noonan/cardio-facio-cutaneous syndrome are shown in dark grey. The grey ovals and light grey oval represent a ligand-bound tyrosine kinase receptor. The mutations shown result in overactivation of the Ras-signalling pathway. SHP-2 = Src homology-2-containing tyrosine phosphatase; GAB = Grb-associated binding protein; Shc = SHC (SrC homology-2 domain-containing)-adaptor protein; GRB2 = growth factor receptor-bound protein-2; SOS1 = Son-ofSevenless-1; GEFs = guanine nucleotide exchanging factors; GAPs = GTPase-activating proteins; RAF = raf oncogen; MAPK = mitogen-activated protein kinase; ERK = extracellular regulated MAP kinase. Figure adapted from Gelb et al. [11].

\section{New paradigms \\ Long-range gene regulation}

\section{Locus control region transcription plays an active role in long-range gene activation}

Ho Y, Elefant F, Liebhaber SA, Cooke NE

Department of Genetics, University of Pennsylvania School of Medicine, Philadelphia, Pa., USA

necooke@mail.med.upenn.edu

Mol Cell 2006:23:365-375

Background: Remote chromatin determinants can regulate the activation of eukaryotic genes such as the $h G H$ gene, which is activated by a $5^{\prime}$-remote locus control region (LCR). Pituitary-specific DNase I hypersensitive site I (HSI) is the dominant $b G H$ LCR element, and is separated from the $b G H$-N promoter by a $14.5-\mathrm{kb}$ region that contains the B-lymphocyte-specific CD79b gene.

Results: Site-specific inactivation of HSI results in loss of acetylation throughout the domain, with a 20 -fold reduction in $h G H-N$ expression. A non-coding domain of Pol II transcription in pituitary somatotropes includes the $b G H$ LCR and adjacent CD79b locus. This entire 'LCR domain of transcription' is HISdependent and terminates $3^{\prime}$ to CD79b. Insertion of a Pol II terminator within the LCR blocks CD79b transcription and represses hGH-N expression. mRNA levels of CD79b are high but not translated.

Conclusion: Removal of HSI reveals a marked repression of transcription throughout the hGH LCR and adjacent CD79b. Thus the hGH LCR is transcribed and the domain of transcription extends to include CD79b. Transcription of the entire region is pituitary-specific and HSI-dependent. The robust HSIdependent LCR domain of transcription is therefore complex - bidirectional $5^{\prime}$ of $C D 79 b$, but in the 'sense' orientation through CD79b. 
Transcription of genes is often regulated by binding of factors to the promoter region, thereby enhancing or repressing transcription of the downstream gene. However, it has become apparent that gene transcription can also be regulated by DNA elements far away from the coding region of the gene. This means that aberrant gene transcription (and thus protein production), for example abnormal GH production, cannot only result from mutations in coding regions of the gene, or mutations in the promoter, but even from mutations in DNA much further upstream or downstream. The region of DNA involved in regulation of transcription of the gene is the 'locus control region' (LCR). This elegant study has given new insights into the role of the LCR in regulating GH expression; the data presented suggest that the LCR and the adjacent gene CD79b are transcribed, and form a domain of transcription. The process is dependent upon the presence of an intact HSI; when this site is inactivated, acetylation of DNA decreases which results in a decrease of both GH and CD79b transcription. Such knowledge of the regulation of the GH gene will help in identifying new causes of aberrant $\mathrm{GH}$ production.

\section{Transactivation function of an $\sim 800$-bp evolutionarily conserved sequence at the SHOX 3' region: implication for the downstream enhancer}

Fukami M, Kato F, Tajima T, Yokoya S, Ogata T

Department of Endocrinology and Metabolism, National Research Institute for Child Health and Development,

Tokyo, Japan

mfukami@nch.go.jp

Am J Hum Genet 2006;78:167-170

Background: The human SHOX gene is one of the major genes contributing to longitudinal growth, and mutations resulting in haploinsufficiency have been reported in patients with isolated short stature and Leri-Weill dyschondrosteosis (LWD). SHOX lies in the pseudoautosomal region (PAR) 1 on both sex chromosomes, and deletions downstream of SHOX have been identified in LWD patients with intact SHOXcoding regions. These data suggest the presence of a downstream enhancer for $S H O X$ transcription.

Methods: Analysis of the SHOX 3' region in five Japanese families in which the proband and one of the parents had variable degrees of LWD in the presence of two copies of intact SHOX-coding exons. The smallest region of overlapping deletion was delineated using the Japanese patients as well as the previously published patients, and within this region, the presence of evolutionarily conserved sequences (ECS) was investigated. Transcriptional activity of the ECS was examined using the human SHOX promoter on exon 2 in a dual-luciferase reporter assay system.

Results: Seven ECS regions were identified, but luciferase activity increased only when ECS4 was cotransfected with the $S H O X$ promoter.

Conclusion: The results suggest that the $\sim 800$-bp ECS4 harbors the putative downstream enhancer for SHOX transcription.

\section{Long-range conserved non-coding SHOX sequences regulate expression in developing chicken limb and are associated with short stature phenotypes in human patients}

Sabherwal N, Bang F, Roth R, Weiss B, Jantz K, Tiecke E, Hinkel GK, Spaich C, Hauffa BP, van der Kemp H, Kapelier J, Tickle C, Rappold G

Department of Molecular Human Genetics, University of Heidelberg, Heidelberg, Germany

gudrun_rappold@med.uni-heidelberg.de

Hum Mol Genet 2007;16:210-222

Background: Deletions downstream of SHOX have been identified in patients with Leri-Weill dyschondrosteosis (LWD) with intact $S H O X$-coding regions. These data suggest the presence of a downstream enhancer for $S H O X$ transcription.

Methods: Analysis of four families with LWD with deletions in the pseudoautosomal region of the sex chromosomes, but with an intact SHOX-coding region, using the techniques of fluorescence in situ hybridization, single nucleotide polymorphism analysis, and comparative genomic analysis. Enhancer potential of candidate regions was performed in chicken embryos by in ovo electroporation of the limb bud, using a green fluorescent protein reporter construct driven by the $\beta$-globin promoter. 
Results: An interval of $\sim 200 \mathrm{~kb}$ that was deleted in all tested affected family members but retained in unaffected members as well as 100 control individuals was identified. Eight highly conserved non-genic elements were identified between 48 and $215 \mathrm{~kb}$ downstream of the SHOX gene. Of these, cis-regulatory activity was observed in three elements in the developing limbs.

Conclusion: These data suggest that the deleted regions in these families contain several distinct elements that regulate $S H O X$ expression in the developing limb. The deletions are associated with a phenotype that is apparently indistinguishable from those patients with mutations in the SHOX-coding region.

This paper is similar to the work by Ho et al. in that it concerns regulation of gene transcription by pieces of DNA a long distance away from the coding region of the gene. These two studies suggest that deletions of downstream enhancers of SHOX may account for some short stature phenotypes. The incidence of SHOX mutations/deletions accounts for around 50-70\% of patients with LWD, and approximately $3-15 \%$ of patients with idiopathic short stature. It appears likely that a further percentage of these phenotypes might be accounted for by mutations/deletions of downstream enhancers of SHOX. These data have wider implications for other monogenic disorders, disorders for which one gene mutation is responsible, as well; mutations should not only be sought in coding regions of DNA or gene promoters, but also in enhancers or repressors further up- or downstream of the gene.

\section{New hope \\ Correction of abnormal IGF-2 imprinting}

\section{Correction of aberrant imprinting of IGF-2 in human tumors by nuclear transfer-induced epigenetic reprogramming}

Chen HL, Li T, Qiu XW, Wu J, Ling JQ, Sun ZH, Wang W, Chen W, Hou A, Vu TH, Hoffman AR, Hu JF

Medical Service, VA Palo Alto Health Care System, Palo Alto, Calif., USA

jifan@stanford.edu

EMBO J 2006:25:5329-5338

Background: Loss of genomic imprinting of insulin-like growth factor-2 (IGF-2) is a hallmark of many human neoplasms. The authors aimed to correct this aberrant epigenotype in tumor cells.

Methods and Results: Nuclei from human tumor cells that showed loss of IGF-2 imprinting were transferred into enucleated mouse and human fibroblasts that had maintained normal IGF-2 imprinting. After nuclear transfer, the abnormal biallelic expression of IGF-2 in tumor nuclei transiently converted to normal monoallelic imprinted expression in the reconstructed diploid cells. In tetraploid hybrid cells normal IGF-2 imprinting was permanently restored in the tumor genome. Cyclohexamide inhibits the synthesis of putative trans-imprinting factors and led to loss of IGF-2 imprinting in normal cultured fibroblasts, suggesting that normal cells produce proteins that act in trans to induce or maintain genomic imprinting.

Conclusion: Abnormal tumor epigenotype can be corrected by in vitro reprogramming, suggesting that loss of imprinting is associated with the loss of activity of trans-imprinting factor(s) that are either inactivated or mutated in tumors.

Loss of genomic imprinting of IGF-2 is a hallmark of many human neoplasms. In theory, correction of this aberrant epigenotype should be possible. Genomic imprinting is the feature of genomes in which only one set of a pair of genes present on homologous chromosomes is expressed, the second gene being silenced by methylation. Additionally, methylation of imprinting control regions can control transcription. IGF-2 is an imprinted gene that is expressed only from the paternal allele in a tissue, promoter- and development-specific manner. Loss of IGF-2 imprinting results in biallelic expression and abnormally high IGF-2 production, and has been found in many human neoplasms, for example in Wilms' tumor. Control of IGF-2 imprinting is complex and in this paper the authors tried to normalize IGF-2 imprinting in tumor cells. Tumor cells with loss of IGF-2 imprinting were used and their nuclei were transferred to normal cells that had their nuclei removed. This resulted in normalization of IGF-2 imprinting so that IGF-2 was only transcribed from one gene; however, this effect was only 
transient and DNA methylation was not altered. The authors speculated that this was the case because enzymes necessary for genomic imprinting were not being produced by the nuclei from the tumor cells. In the next experiment therefore, hybrid cells were made by fusing tumor cells with normal cells. In these hybrid tetraploid cells, IGF-2 imprinting was normalized and monoallelic IGF-2 expression was permanent and the authors suggest that the presence of cytoplasmic active 'transimprinting' factors are involved in the maintenance of normal imprinting. This work is an important step in understanding and altering abnormal gene imprinting in tumor formation.

\title{
New animal models \\ GH action
}

\section{In vivo imaging of hepatic GH signalling}

\author{
Frank S, Wang X, He K, Yang N, Fang P, Rosenfeld RG, Hwa V, Chaudhuri TR, Deng L, Zinn KR \\ Department of Medicine, University of Alabama at Birmingham, Ala., USA \\ sjfrank@uab.edu
}

Mol Endocrinol 2006;20:2819-2830

Background: This study aimed to develop a mouse model system to non-invasively and repeatedly image in vivo hepatic GH signalling.

Methods: Nude mice were used for adenoviral mediated delivery of STAT5-dependent GH response element luciferase reporter to detect GH signalling serially by bioluminescence imaging.

Results: Female nude mice were injected with Ad-GHRE-luc and 3 days later fasted for $16 \mathrm{~h}$. They were then anesthetized and injected with i.p. luciferin for a baseline image, and then injected with i.v. hGH. They received another injection of i.p. luciferin 1, 3, 5 and $7 \mathrm{~h}$ later. Bioluminescence images were collected $10 \mathrm{~min}$ after each luciferin injection. Luminescence was detected $1 \mathrm{~h}$ after hGH injection and was most intense $3 \mathrm{~h}$ after hGH stimulation, and was mostly in the region of the liver.

Conclusion: This system allows for in vivo analysis of Stat5-dependent signalling.

Luciferase reporters have been used for many years to quantify gene activation in vitro, but Frank et al. have generated a system to detect and quantify Stat5-induced luciferase activity in live animals. They created an adenoviral vector composed of the firefly luciferase gene driven by a promoter with eight repeats of the STAT5-dependent GH-response element of Spi2.1 (Ad-GHRE-luc) and injected it into nude mice. Adenovirus primarily targets the liver and therefore vector injection should result in enhanced expression of the reporter in the liver. Using a tail vein injected HA-labelled GHR adenoviral vector and in vivo injected radioactive antibodies to $\mathrm{HA}$, the authors very elegantly showed that indeed the adenoviral vector mainly targeted the liver. Once the authors had established this, they continued to visualize the response to $\mathrm{hGH}$. To do this, female mice were injected with the Ad-GHREluciferase vector. Stimulation by $\mathrm{GH}$ will result in Stat5 production and activation, which will then bind to the GHRE-luciferase DNA and as a result luciferase will be transcribed. The injected luciferin will give rise to a bioluminescence signal, which can be measured and is dependent on the amount of luciferase produced. The authors used a sensitive imaging system that was able to measure the bioluminescence signal in live animals. The authors also found that fasting increased the sensitivity to exogenous GH when mice received adenoviral GHRE-Luc in combination with adenoviral GHR. In conclusion, this is a very useful system for the in vivo analysis of cytokine induced Stat5 signalling that may also be used in other rodent models and will increase our understanding of the regulation of GH signalling. 


\section{Elevated circulating insulin-like growth factor-binding protein-1 is sufficient to cause fetal growth restriction}

Watson CS, Bialek P, Anzo M, Khosravi J, Yee S-P, Han VKM

Samuel Lunenfeld Research Institute, Mount Sinai Hospital, Toronto, Ont., Canada

watson@mshri.on.ca

Endocrinology 2006;147:1175-1186

Background: Circulating IGF-binding protein-1 (IGFBP-1) is elevated in newborns and experimental animals with fetal growth restriction (FGR) and it is known that IGFBP-1 can inhibit actions of IGF-1. The aim of this study was to study a possible causal relationship between high circulating IGFBP-1 and FGR. Methods: Transgenic mice overexpressing human IGFBP-1 (hIGFBP-1) driven by mouse $\alpha$-fetoprotein gene promoter in the fetal liver were generated.

Results: Transgenic mice (AFP-BP-1) expressed hIGFBP-1 mainly in the fetal hepatocytes, starting at embryonic day 14.5 (E14.5) and peaking at 1 week postnatally. At birth, AFP-BP-1 pups were $18 \%$ smaller, and mice did not demonstrate any postnatal catch-up growth. The placentas of the AFP-BP-1 mice were larger than WT from E16.5 onwards.

Conclusion: High concentrations of circulating IGFBP-1 are sufficient to cause FGR.

\section{Insulin-like growth factor-binding protein (IGFBP-1) involvement in intrauterine growth retardation: study on IGFBP-1 overexpressing transgenic mice}

Ben Lagha N, Seurin D, Le Bouc Y, Binoux M, Berdal A, Menuelle P, Babajko S

Laboratoire de Biologie Oro-faciale et Pathologie, INSERM Unit 714, Institut Biomédical des Cordeliers, Paris, France

Sylvie.Babajko@bhdc.jussieu.fr

Endocrinology 2006; 147:4730-4737

Background: In this work, the authors wished to establish the impact of circulating IGFBP-1 on body growth associated to bone mineralization and carbohydrate resources.

Methods: Transgenic mice used in this work overexpressed human IGFBP-1, driven from the human $\alpha_{1}$-antitrypsin promoter, in liver from embryonic day (E)14.5, concomitantly to the appearance of ossification centers, through to adulthood.

Results: Growth retardation was observed as early as E17.5 in homozygous mice, which were $20 \%$ smaller at birth. The mice exhibited pleiotropic defects of several skeletal units in the appendicular and axial skeleton. IGFBP-1 overexpression contributed to decreased fetal hepatic glycogen and neonatal circulating glucose levels.

Conclusion: Overexpression of fetal human IGFBP-1 is related to antenatal growth retardation and delayed bone mineralization in transgenic mice.

There is a large body of evidence from animal experiments and clinical observation implicating the IGF system in modulating fetal growth. Both the ligand (IGF-1) and receptor are involved in the process. Less is known of the role for the IGF-binding proteins in the modulation of fetal growth. These two complementary papers demonstrate that animals in which IGFBP-1 has been overexpressed are $18-20 \%$ smaller than the wild-type animals. Altered bone size and mineralization accompanied the loss of IGFBP-1 along with a reduction in fetal hepatic glycogen and blood glucose concentration. Of interest was the observation that placental size was increased although the components of the placenta responsible for this change were not characterized. Postnatal growth was normal in both overexpressing and wild-type animals so catch-up growth was not observed. 


\title{
Codependence of growth hormone-responsive, sexually dimorphic hepatic gene expression on signal transducer and activator of transcription $5 \mathbf{b}$ and hepatic nuclear factor $\mathbf{4 \alpha}$
}

Holloway MG, Lax EV, Waxman DJ

Division of Cell and Molecular Biology, Boston University, Boston, Mass., USA

djw@bu.edu

Mol Endocrinol 2006;20:647-660

Background: Stat5 deficiency results in decreased expression in male mouse liver of male-predominant cytochrome $\mathrm{P}_{450}$ CYP2d enzymes and an increase of female-predominant Cyp2b proteins.

Methods: This study characterized the effects of Stat5b deficiency on 15 individual Cyp RNAs, and assessed the effect of Stat 5 b deficiency, HNF $4 \alpha$ deficiency and GH regulation (by hypophysectomy, pulsatile GH treatment, continuous GH treatment).

Results and Conclusion: All 7 male-specific RNAs were decreased to female levels in Stat5b-deficient male liver, whereas 5 of 8 female-specific RNAs (designated class I) were increased in expression up to 200fold. Stat $5 \mathrm{~b}$ deficiency had a much more modest effect on the expression of these genes in females. The female-specific genes could be designated to two groups: class I mRNAs that were affected by Stat $5 \mathrm{~b}$ deficiency and class II mRNAs that were not affected by Stat5b deficiency. Hypophysectomy and GH treatment studies showed positive GH pulse regulation of all 7 male RNAs and negative GH pulse regulation of class I but not class II female RNAs in male mice. Many of the gender-specific genes responded in parallel to the loss of Stat $5 b$ and the loss of HNF4 $\alpha$ suggesting that Stat5b and HNF $4 \alpha$ may coregulate gender-specific gene expression. Continuous GH treatment of intact male mice induced expression of class I female RNAs in 4-7 days but of class II RNAs not until 7-14 days. Given the slow response of all 15 genes to changes in $\mathrm{GH}$ status, the authors propose that regulation of gender-specific CYP expression is indirect and mediated by Stat $5 b$ - and HNF4 $\alpha$-dependent factors that may include repressors of female-specific Cyps and other targets of GH action.

\section{Sex-dependent liver gene expression is extensive and largely dependent upon signal transducer and activator of transcription 5b (Stat5b): Stat5b-dependent activation of male genes and repression of female genes revealed by microarray analysis}

\author{
Clodfelter KH, Holloway MG, Hodor P, Park S-H, Ray WJ, Waxman DJ \\ Division of Cell and Molecular Biology, Boston University, Boston, Mass., USA \\ djw@bu.edu \\ Mol Endocrinol 2006;20:1333-1351
}

Background: Sexual dimorphism in mammalian liver contributes to gender differences in physiology and many sex-dependent liver genes are regulated by GH and Stat $5 \mathrm{~b}$.

Methods: A large-scale gene expression study was conducted to characterize sex differences in liver gene expression and their dependence on Stat5b. Male and female, wild-type and Stat5b-deficient mice were used.

Results: 850 genes were more highly expressed in males and $90 \%$ were decreased in Stat 5 b deficiency. 753 genes were female-predominant of which $61 \%$ were upregulated in Stat $5 \mathrm{~b}$-deficient males. However, $90 \%$ of the gender-dependent genes were unaffected by Stat $5 \mathrm{~b}$ deficiency in females.

Conclusion: Stat $5 \mathrm{~b}$ is essential for sex-dependent liver gene expression, equalling $4 \%$ of the genome. Malepredominant liver gene expression requires Stat $5 \mathrm{~b}$ or Stat $5 \mathrm{~b}$-dependent factors. Many female-predominant liver genes are repressed in males in a Stat $5 b$-dependent manner.

The main signalling transduction pathway for GH is the JAK2-STAT5 pathway and activation of this pathway is differentially affected by 'male' and 'female' GH secretory patterns (fig. 2). A pulsatile 'male-like' GH pattern stimulates Stat5b activation to a greater extent than a more continuous 'female-like' GH pattern. Stat5b-deleted mice are small, especially the males, and have altered hepatic 


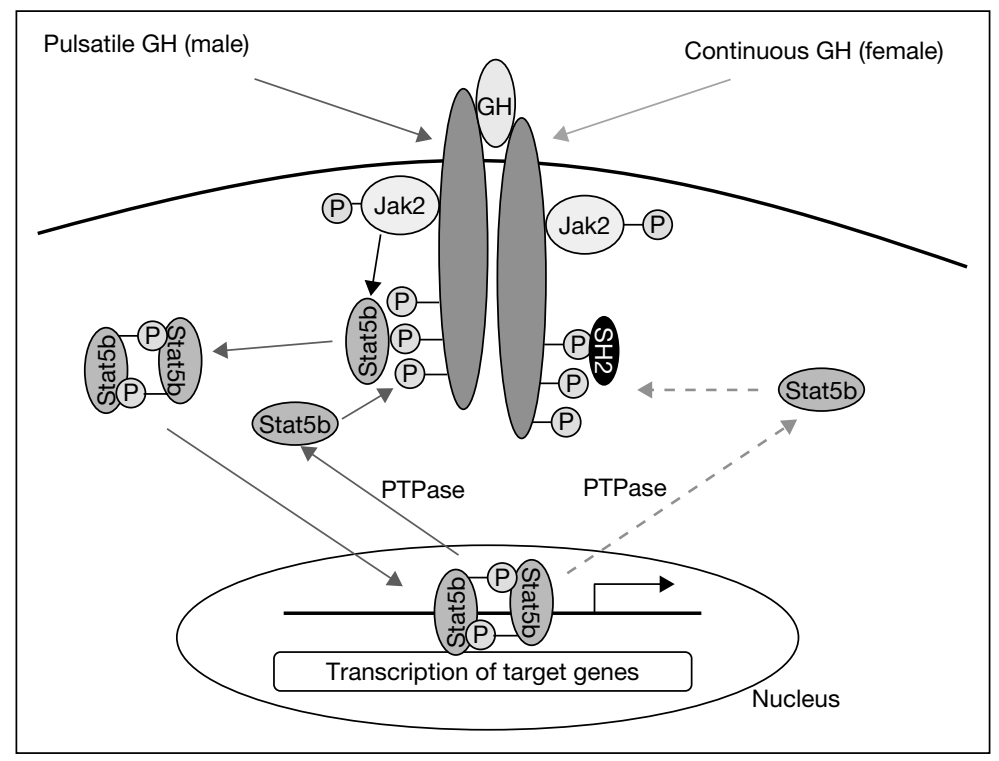

Fig. 2. Sexual dimorphism in $\mathrm{GH}$-activated Stat5b phosphorylation. GH binding to dimerized GH receptors (GHR) results in proximity of GHR-associated tyrosine kinase JAK2 molecules, resulting in autophosphorylation and tyrosine phosphorylation of GHR residues, generating docking sites for Stat5b and other SH2 domain-containing proteins. After binding to these sites, Stat5b undergoes JAK2 catalyzed tyrosine phosphorylation, followed by dimerization, nuclear translocation and induction of transcription of target genes. Stat $5 b$ is then deactivated by phosphotyrosine phosphatases (PTPase), and Stat5b may then be reactivated in subsequent cycles of docking and phosphorylation. In females, the Stat $5 b$ is much less activated and the Stat5b cycle is more rapidly terminated. Jak2 = Janus kinase 2; $P=$ phosphate; $\mathrm{Stat} 5 \mathrm{~b}=$ signal transducer and activator of transcription 5b; PTPase = phosphotyrosine phosphatase; $\mathrm{SH} 2=\mathrm{Scr}$ homology-2-containing protein. Figure redrawn from Waxman and O'Connor [4].

gene expression. The papers above describe two extensive studies regarding the regulation of genderspecific hepatic gene expression. Many of these genes were found to be affected by Stat5b deficiency in males, but not necessarily in females. Several of the Stat5b-dependent male genes encode transcriptional repressors; these may include direct Stat5b targets that repress female-predominant genes in male liver. Several female-predominant repressors were elevated in Stat5b-deficient males; these may contribute to the major loss of male gene expression seen in the absence of Stat $5 \mathrm{~b}$. The involvement of Stat $5 b$ in the regulation of sex-dependent genes is therefore not straightforward. HNF4 $\alpha$ also contributes to gender specificity of liver gene expression through positive regulation of male-specific Cyp genes and negative regulation of female-specific Cyp genes. The authors showed that many genes are co-dependent on Stat5b and HNF4 $\alpha$ and suggest that Stat5b and HNF4 $\alpha$ act in concert, by an indirect mechanism, to affect expression of sexually dimorphic hepatic genes. Figure 3 shows a hypothesis of gender-specific hepatic gene regulation based on these findings. Since $4 \%$ of the genome is affected by Stat5b deficiency, responsible for gender differences in fat, carbohydrate, steroid and drug metabolism, it is important to elucidate underlying mechanisms and these studies help in doing so. An excellent review by Waxman and O'Connor [4] discusses this matter further. 


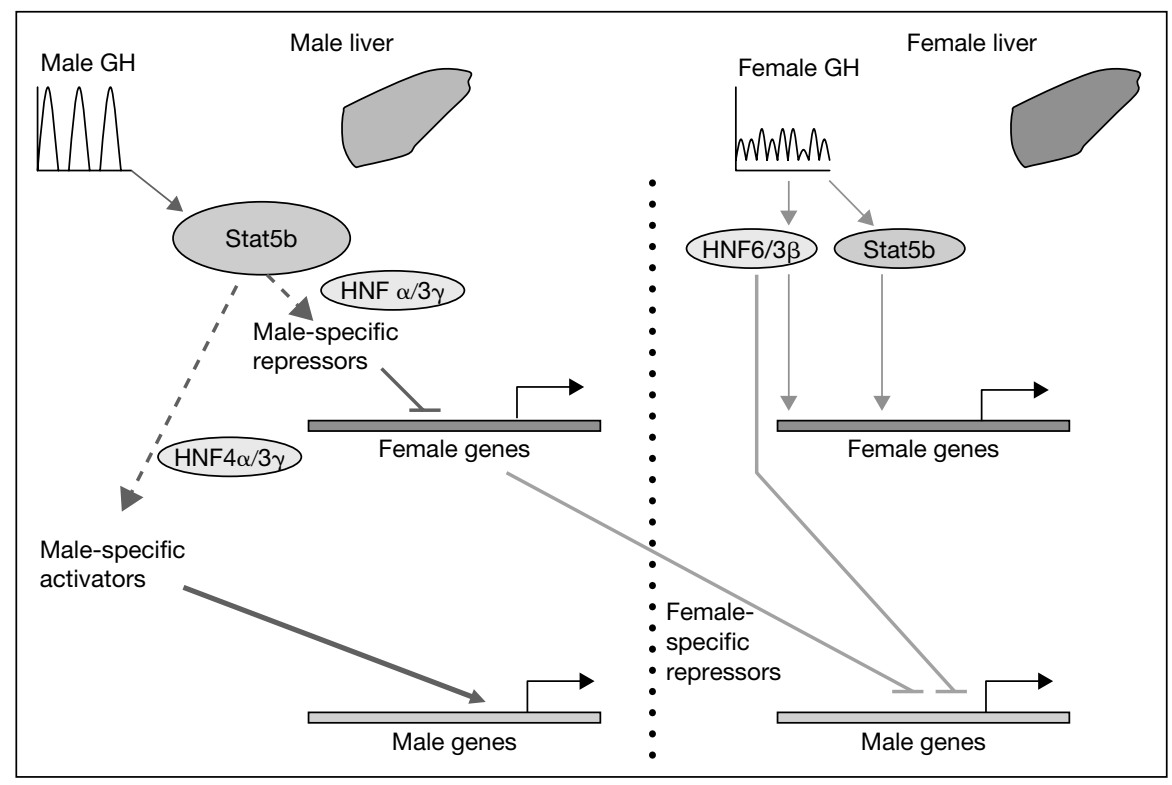

Fig. 3. Hypothetical mechanism for indirect regulation of sex-specific genes by liver Stat5b and hepatic nucleic factors (HNFs). Male GH pulse-activated Stat5b is proposed to activate male-specific activators and male-specific repressors, which then respectively induce male-specific genes and repress female-specific genes. The female-specific genes will include female repressors that inhibit the expression of male-specific genes in female liver. The low Stat5 activity found in female liver may act directly or indirectly (via HNF6 and/or HNF3ß) to induce female gene expression. HNF6 can inhibit transcription of male-specific genes. HNF4 $\alpha$ is required for transcription of a subset of Stat5b induced male-specific genes, as observed in the co-dependence of gender-specific gene expression on Stat5b and HNF4 $\alpha$. Figure adapted from Waxman and O'Connor [4].

\section{Continuing debate Liver-produced IGF-1}

\section{Liver-specific overexpression of the insulin-like growth factor-1 enhances somatic growth and partially prevents the effects of growth hormone deficiency}

Liao L, Dearth RK, Zhou S, Britton OL, Lee AV, Xu J

Department of Molecular and Cellular Biology, Baylor College of Medicine, Houston, Tex., USA

jxu@bcm.tmc.edu

Endocrinology 2006;147:3877-3888

Background: Since the role and importance of circulating IGF-1 in body growth remains unclear, the authors wished to create an animal model that secreted supraphysiological amounts of IGF-1 from the liver. Methods: Mice carrying a transthyretin enhancer-promoter-IGF-1 transgene were created.

Results: Mice transcribed transgene IGF-1 solely in the liver and had a 50-60\% increase in circulating IGF-1 levels. Transgenic (TG) mice had increased body weight and body length compared with wildtype (WT) mice. Lean body mass was increased due to increased number and thickness of skeletal muscle fibers. Treatment with GH antagonist Pegvisomant caused a severe growth deficit in WT mice but Pegvisomant also reduced body growth in TG mice. Glucose tolerance was slightly improved in TG mice compared to WT mice.

Conclusion: Higher circulating levels of IGF-1 stimulate somatic growth and lean body mass modestly and improve glucose tolerance slightly. 
This is a nice study in which the authors created a mouse strain that could be seen as the mirror model of the liver induced IGF-1-deficient (LID) mice. LID mice have a $75 \%$ reduction in circulating IGF-1 levels but normal growth, suggesting that liver-generated IGF-1 does not play a major role in the promotion of growth, although several aspects of this model have been criticized $[5,6]$. The mouse model created by Liao et al. has a liver-specific overexpression of mouse IGF-1 resulting in a modest $50-60 \%$ increase in circulating IGF-1 levels. These mice have a modestly increased body weight of ca. $10 \%$ and bone length (5\%) that reaches statistical significance at the age of 8-9 weeks. This suggests that supraphysiological IGF-1 production is able to increase growth modestly, in line with response to IGF-1 treatment in GHD rodents and humans. The increase in muscle mass in these mice was much more impressive, with a $30 \%$ increase in soleus thickness, muscle fiber number and fiber thickness. Blockade of GH signalling with Pegvisomant (from 21 to 56 days of age) resulted in a reduction of body growth in both TG and WT mice to a similar extent. Although transgene IGF-1 expression was unaffected by Pegvisomant, reduction of endogenous IGF-1 transcription and reduction of ALS production resulted in a $50 \%$ reduction of IGF-1 levels in the TG mice. Therefore, the mechanism of GHR blockade-induced reduction of growth is still unclear and can be due to either a blockade of direct GH action in peripheral tissues or reduced stimulation by IGF-1. Cross-breeding of these mice with mice harboring other defects in the GH-IGF-1 axis may take us a step further.

\section{Important for clinical practice Small children growing up}

\section{Growth trajectories of extremely low birth weight infants from birth to young adulthood: a longitudinal, population-based study}

Saigal S, Stoskopf B, Streiner D, Paneth N, Pinelli J, Boyle M

Department of Pediatrics, McMaster University, Hamilton, Ont., Canada

saigal@mcmaster.ca

Pediatr Res 2006;60:751-758

Background: The outcome of growth attainment of children born with extremely low birth weight (ELBW) is not well known and the authors therefore aimed to study this in a population-based cohort.

Methods: Growth attainment of a population-based cohort of ELBW $(<1,000 \mathrm{~g})$ and a cohort of sociodemographically comparable normal birth weight (NBW) was compared at young adulthood, and the pattern of growth trajectories and correlates of growth at ages 1,2, 3, and 8 years, and teen and young adulthood were compared.

Results: The proportion considered small for gestational age was ELBW 25\% versus NBW 3\%. Weight for age z-scores for ELBW showed substantial decline to age 3 years, with subsequent catch up to adolescence and smaller gains to adulthood.

Conclusion: ELBW children showed growth failure during infancy, followed by accelerated weight gain and crossing of BMI percentiles at adolescence.

Long-term growth outcomes for ELBW infants are not common, so this comparison from a population of 147 ELBW $(<1,000 \mathrm{~g})$ and 131 sociodemographically comparable normal birth weight (NBW) cases at young adulthood is of value. Weight for age z-scores for ELBW showed substantial decline to age 3 years, with subsequent significant catch up to adolescence and smaller gains to adulthood. Height for age z-scores showed both sexes of ELBW were disadvantaged at every age compared with NBW and their expected mid-parental height. The BMI z-scores for ELBW showed a sustained incline from age 3 years to adulthood, where both sexes normalized to above zero, and were comparable to their peers. These patterns of growth may be particularly important in the ELBW children, as in other studies it has been associated with an increased risk of insulin resistance and coronary heart disease. 


\section{The influence of head growth in fetal life, infancy, and childhood on intelligence at the ages of 4 and 8 years}

Gale CR, O'Callaghan FJ, Bredow M, Martyn CN

Medical Research Council Epidemiology Resource Centre, University of Southampton, Southampton, UK

crg@mrc.soton.ac.uk

Pediatrics 2006;118:1486-1492

Background: The relation between head growth and cognitive function is not clear and in this work, the authors studied the effects of head growth prenatally, during infancy, and during later periods of development on cognitive function at the ages of 4 and 8 years.

Methods: 633 term-born children from the Avon Longitudinal Study of Parents and Children cohort were followed and their cognitive function was assessed at the age of 4 and 8 years.

Results: At 4 years, full-scale IQ increased by an average of 2.41 points for each 1 SD increase in head circumference at birth and 1.97 points for each 1 SD increase in head growth during infancy, conditional on head size at birth. At 8 years, head circumference at birth was no longer associated with IQ, but head growth during infancy remained a significant predictor, with full-scale IQ increasing an average of 1.56 points for each $1 \mathrm{SD}$ increase in growth.

Conclusion: This study suggests that the brain volume a child achieves by the age of 1 year helps determine later intelligence. Growth in brain volume after infancy may not compensate for poorer earlier growth.

\section{Impact of prenatal and/or postnatal growth problems in low birth weight preterm infants on school-age outcomes: an 8-year longitudinal evaluation}

Casey PH, Whiteside-Mansell L, Barrett K, Bradley RH, Gargus R

Department of Pediatrics, University of Arkansas for Medical Sciences, Little Rock, Ark., USA

CaseyPatrickH@uams.edu

Pediatrics 2006;118:1078-1086

Background: The objective of this study was to assess the 8-year growth, cognitive, behavioral status, health status, and academic achievement in low birth weight preterm infants who had failure to thrive only, were small for gestational age only, had failure to thrive plus were small for gestational age, or had normal growth.

Methods: A total of 985 infants were evaluated until age 8; 180 infants met the criteria for failure to thrive between 4 and 36 months' gestational corrected age.

Conclusion: Low birth weight preterm infants who had postnatal growth problems, particularly when associated with prenatal growth problems, demonstrated lower physical size, cognitive scores, and academic achievement at age 8 . When postnatal growth was adequate, there was no independent effect of small for gestational age status on cognitive status and academic achievement.

Poor head growth occurs in a number of situations, particularly in severe intrauterine growth restriction and in the babies of mothers who smoke during pregnancy. These two papers demonstrate that (Gale et al.) when the influence of head growth was distinguished for different periods, only prenatal growth and growth during infancy were associated with subsequent IQ. These observations pertain to the general population but are largely echoed in the study (Casey et al.) in low birth weight infants assessed at the age of 8 years. In this situation, children who were both small for gestational age and had failure to thrive were the smallest in all growth variables at age 8 , and also demonstrated the lowest cognitive and academic achievement scores. The children with failure to thrive only were significantly smaller than the children with normal growth in all growth variables and had significantly lower IQ scores. Those who were small for gestational age only did not differ from those with normal growth in any cognitive or academic achievement measures. These observations suggest that the brain volume a child achieves by the age of 1 year helps determine later intelligence and growth in brain volume after infancy may not compensate for poorer earlier growth particularly in situations where postnatal growth is also compromised. 


\section{Evidence for hypermetabolism in boys with constitutional delay of growth and maturation}

Han JC, Balagopal P, Sweeten S, Darmaun D, Mauras N

Division of Endocrinology, Nemours Children's, Jacksonville, Fla., USA

nmauras@nemours.org

J Clin Endocrinol Metab 2006;91:2081-2086

Background: The authors hypothesized that an imbalance between energy intake and expenditure may contribute to the pathogenesis of constitutional delay of growth and maturation (CDGM) and therefore compared differences in nutrition, body composition and energy expenditure in boys with CDGM and controls.

Methods: Observational, cross-sectional study of 36 boys (8-17 years): 12 with CDGM (short stature, delayed bone age and puberty, and no other pathology) and 12 height-matched (pre- or early pubertal) and 12 age-matched (pubertal) healthy controls.

Results: Nutritional markers were comparable among the groups. CDGM subjects had bone mineral density lower than age-matched controls $(\mathrm{p}<0.01)$ but comparable with height-matched controls. Even though resting energy expenditure did not differ between groups, CDGM subjects had $25 \%$ higher caloric intake adjusted for fat-free mass (FFM) than height-matched controls $(\mathrm{p}<0.05)$ and $78 \%$ higher caloric intake per kilogram FFM compared with age-matched controls $(\mathrm{p}<0.00001)$. CDGM subjects had $46 \%(\mathrm{p}<0.05)$ and $91 \%(\mathrm{p}<0.001)$ higher total energy expenditure per kilogram FFM than height- and age-matched controls, respectively.

Conclusions: Boys with CDGM have higher rates of overall energy expenditure compared with age- and size-matched controls. This increased metabolism may result in impaired tempo of growth.

Children with CDGM tend to be thin and it has been suggested their growth pattern is reminiscent of nutritional insufficiency. Using doubly labelled water studies, serum nutritional/hormonal markers, dual-energy x-ray absorptiometry, dietary analysis, and indirect calorimetry values were compared between patients with CDGM and height- or age-matched controls. Even though resting energy expenditure did not differ between groups, CDGM subjects had $25 \%$ higher caloric intake adjusted for FFM than height-matched controls and $78 \%$ higher caloric intake per kilogram FFM compared with age-matched controls. IGF-1 and testosterone were by definition lower than age-matched controls. Although it was suggested that augmenting nutrition to match energy needs (with or without hormonal therapy) might improve linear and ponderal growth, given that adult outcomes for this group of individuals is no different from the general population, it is difficult to see the advantage of such an approach.

Clinical trials, new treatment

\section{Growth hormone is effective in treatment of short stature associated with short stature homeobox-containing gene deficiency: two-year results of a randomized, controlled, multicenter trial}

Blum WF, Crowe BJ, Quigley CA, Jung H, Cao D, Ross JL, Braun L, Rappold GJ

Lilly Research Laboratories, Eli Lilly \& Co., Bad Homburg, Germany

Blum_Werner@Lilly.com

J Clin Endocrinol Metab 2007;92:219-228

Background: The human SHOX gene is one of the major genes contributing to longitudinal growth, and mutations resulting in haploinsufficiency have been reported in patients with isolated short stature and Leri-Weill syndrome (LWS). The aim of this study was to determine the efficacy of GH in treating short stature associated with SHOX deficiency (SHOX-D).

Methods: Randomized trial with a GH-treated and untreated group of patients with short stature and proven SHOX-D. Comparisons were made with a GH-treated group of patients with Turner syndrome. 1,608 samples from children with idiopathic short stature or LWS were analyzed for abnormalities of 
the SHOX gene. First- and second-year height velocity, height SD score and height gain $(\mathrm{cm})$ were compared between the groups.

Results: The GH-treated SHOX-D group had a significantly greater first-year height velocity than the untreated control group (mean \pm SE: $8.7 \pm 0.3$ vs. $5.2 \pm 0.2 \mathrm{~cm} /$ year; $\mathrm{p}<0.001$ ) and similar first-year height velocity to $\mathrm{GH}$-treated subjects with TS $(8.9 \pm 0.4 \mathrm{~cm} /$ year; $\mathrm{p}=0.592)$. Second-year height velocity $(7.3 \pm 0.2$; vs. $5.4 \pm 0.2 \mathrm{~cm} /$ year; $\mathrm{p}<0.001)$ and second-year height SDS $(-2.1 \pm 0.2$ vs. $-3 \pm 0.2 ; \mathrm{p}<0.001)$ were all significantly greater in GH-treated subjects than controls and were comparable to second-year height velocity $(7.0 \pm 0.2 \mathrm{~cm} /$ year $)$ and second-year height SDS $(-2.6 \pm 0.2)$ in the GH-treated girls with Turner syndrome.

Conclusion: GH treatment increased height velocity and height SDS over a 2-year treatment period in patients with SHOX-D, and this effect was similar to that observed in TS.

This is a good study size with 52 prepubertal patients with short stature (height $<3$ rd percentile or $<10$ th percentile and $\mathrm{HV}<25$ th percentile) and a molecularly proven SHOX gene defect that were either treated with hGH or not. SHOX gene abnormalities were found in 67 of 1,608 patients with ISS or LWS, and comprised gene deletions, partial gene deletions and point mutations, and randomization was stratified by these different gene abnormalities, the presence of LWS and gender. SHOX-D is believed to contribute significantly to the growth retardation associated with Turner syndrome and therefore comparison was also made with 26 patients with Turner syndrome treated with $\mathrm{GH}$. All patients were treated for 2 years with a dose of $50 \mu \mathrm{g} / \mathrm{kg} /$ day (equalling ca. $1.3 \mathrm{mg} / \mathrm{m}^{2} /$ day). This study shows the beneficial effects of GH treatment in SHOX-D patients treated with GH over a 2-year period. The height SDS improvement was approximately +1 SDS in 2 years and was comparable to the benefit of GH in Turner syndrome. It is therefore not unlikely that final height improvements will be in a similar range as those found in Turner syndrome. Interestingly, the effect of GH treatment seemed somewhat larger in ISS compared to LWS, but it is unclear from this work whether that is due to genotype differences or whether end-organ response to GH treatment is different between these groups.

\section{New mechanisms}

GH action and regulation of signalling

\section{Growth hormone promotes skeletal muscle cell fusion independent of insulin-like growth factor-1 upregulation}

Sotiropoulos A, Ohanna M, Kedzia C, Menon RK, Kopchick JJ, Kelly PA, Pende M

INSERM, Faculté de Médicine Necker-Enfants Malades, Paris, France

sotiropoulos@necker.fr

Proc Natl Acad Sci USA 2006;103:7315-7320

Background: The growth retardation of double GHR/IGF-1 mutants is more severe than that observed with single mutants. It is therefore likely that GH exerts specific and direct actions and the aim of this paper was to identify these GH-specific actions on muscle.

Methods: $\mathrm{GHR}^{-1-}$ mice were studied and primary muscle cell cultures from these mice were used.

Results: The mass of skeletal muscles lacking GHR is reduced because of a decrease in myofiber size, whereas myofiber number is normal. GH has no effect on size, proliferation and differentiation of myoblast precursors, but controls the size of differentiated myotubes in a cell-autonomous manner. The GH hypertrophic action leads to increased myonuclear number, suggesting that GH facilitates fusion of myoblasts with nascent myotubes. This action is independent of IGF-1 but depends on NFATC2, a transcription factor that regulates myoblast-myotube fusion. This effect of GH occurs without an increase in local IGF-1 and also without increasing Stat5.

Conclusion: The effects of GH and IGF-1 on muscle are distinct and additive, and rely on different signalling transduction pathways.

It is well established that GHRs are present in many peripheral tissues and indeed also in muscle, but nevertheless the direct effect of GH on these cells remains unclear. In this study the authors aimed to 
unravel GH-specific actions in muscle. GHR deficiency caused a switch from oxidative slow type I fibers to glycolytic fast type II fibers, consistent with an increase in type I fibers in mice overexpressing hGH [3]. GHD however decreased muscle fiber size by $40 \%$ in both type I and type II fibers. It was shown that this was not due to altered proliferation or differentiation of myoblasts but that $\mathrm{GH}$ increases the size of myotubes by promoting fusion of myotubes with myoblasts. Muscle cells are multinucleated cells and muscle hypertrophy occurs through fusion of cells, and this process is stimulated by $\mathrm{GH}$. This is an important paper since it suggests a new concept of the mechanism of GH action.

\section{Functional cross-modulation between SOCS proteins can stimulate cytokine signalling}

Piessevaux J, Lavens D, Montoye T, Wauman J, Catteeuw D, Vandekerckhove J, Belsham D, Peelman F, Tavernier J

Flanders Interuniversity Institute for Biotechnology, Ghent University, Ghent, Belgium

jan.tavernier@ugent.be

J Biol Chem 2006;281:32953-32966

Background: SOCS (suppressors of cytokine signalling) are negative regulators of cytokine signalling that function primarily at the receptor level. Interestingly, SOCS2 can have both inhibitory and stimulatory effects on GH signalling. Other SOCS proteins can also inhibit GH signalling and therefore this study examined direct cross-modulation between SOCS proteins.

Methods: Cell culture, transfection with labelled SOCS constructs, cytokine receptors and luciferase reporters, Western blotting and co-immunoprecipitation.

Results: The authors demonstrated that SOCS2 interfered with the negative regulatory effects of SOCS1 and SOCS3 via direct interaction, possibly due to degradation of the targeted SOCS proteins. SOCS2 can interact with all members of the SOCS family and not only affects GH signalling but also interferon and leptin signalling. These observations were extended to SOCS6 and SOCS7.

Conclusions: SOCS2, SOCS6 and SOCS7 are capable of controlling SOCS protein stability and thus altering cytokine sensitivity.

Negative control of cytokine signalling occurs at many levels and involves, amongst others, inhibition of Stat activation by SOCS proteins. The SH2 domain of SOCS2 inhibits Stat activation by competition with Stats for binding to phosphorylated receptor-docking sites necessary for Stat phosphorylation. In accordance, SOCS2 knockout mice display overgrowth and have increased sensitivity to GH, but interestingly overexpression of SOCS2 also results in gigantism. This study aids in the explanation of the underlying physiological mechanism and shows that SOCS2 plays a pivotal role in the regulation of cytokine sensitivity. Expression of SOCS1 and SOCS3 was able to completely abolish GH signalling in vitro and co-expression of SOCS2 counteracted this effect. This was not only the case for GH signalling but also for IFN- $\gamma$ and leptin signalling. The authors showed that SOCS2 directly interacted with elongin BC complex after which the complex bound to SOCS1 and SOCS3. The elongin BC complex is part of a complex that marks proteins for degradation, and indeed the authors showed that SOCS1 was degraded by increasing levels of SOCS2. These results suggest that SOCS2, but also SOCS6 and SOCS7, regulates multiple cytokine-signalling pathways. So even though SOCS2 is mainly associated with inhibiting GH signalling, it can increase signalling of IFN- $\gamma$ and leptin, and possibly other cytokines, through increased degradation of SOCS1 and SOCS3 that normally inhibit IFN- $\gamma$ and leptin signalling. This may be of help in defining underlying mechanisms for alterations of cytokine signalling in states of GH deficiency, insensitivity or overproduction. 


\section{Clinical and biochemical characteristics of a male patient with a novel homozygous Stat5b mutation}

Vidarsdottir S, Walenkamp MJE, Pereira AM, Karperien M, Van Doorn J, Van Duyvenvoorde HA, White S, Breuning MH, Roelfsema F, Kruithof MF, Van Dissel J, Janssen R, Wit JM, Romijn JA

Department of Endocrinology and Metabolic Diseases, Leiden University Medical Center, Leiden, The Netherlands m.j.e.walenkamp@lumc.nl

J Clin Endocrinol Metab 2006;91:3482-3485

Background: Recently, 2 females with severe short stature and pulmonary and immune abnormalities were found to have homozygous mutations in the $S t a t 5 b$ gene. This paper describes a new Stat $5 b$ mutation in a male with severe short stature.

Methods: Genotyping of Stat5 cDNA and Western blotting for Stat5b in cultured fibroblasts before and after $\mathrm{GH}$ stimulation.

Results: A homozygous frameshift mutation in the DNA-binding domain of Stat $5 b$ gene was found in a patient with severe short stature (-5.9SDS), delayed puberty, and no history of pulmonary or immunological problems. Plasma prolactin was elevated. IGF-1, IGFBP-3 and ALS were extremely low (-6.9, -12 and -7.5 SDS).

Conclusion: This report confirms the essential role of Stat5b in GH signalling and growth in the human, in both males and females, and suggests that pulmonary and immunological abnormalities are not necessarily part of the phenotype.

This is an interesting paper showing that human Stat5b mutations are not confined to the female gender. In mice, Stat5b deficiency affects growth of males more than that of females, possibly related to the more pulsatile fashion of $\mathrm{GH}$ secretion in males. In humans the distinction between genders regarding GH secretion is not as pronounced as it is in rodents and this may underlie the fact that the severity of the growth defect in this male patient is similar to that described in the 2 females. However, a greater number of affected patients are required to assess possible gender differences. The second interesting observation is that this patient lacks pulmonary or immunological pathology. In addition, this patient differs from other patients with Stat5b mutations in that he did not have elevated $\mathrm{GH}$ secretion, even though he did have enhanced prolactin secretion. The pathophysiological mechanism of the pulmonary abnormalities is still unclear but in the patient described by Kofoed et al. [7], Stat1 and Stat3 signalling was increased and this may contribute to altered immune function and we do not know whether that is the case in this patient. We now know of 2 more patients (siblings) with a Stat5b mutation (a homozygous splice site mutation in exon 13) who do not suffer from pulmonary disease but 1 of whom has juvenile idiopathic arthritis, also suggesting disordered immune function [8].

\section{Novel mutations in known genes: acid-labile subunit}

\section{Total absence of functional-acid labile subunit, resulting in severe insulin-like growth factor deficiency and moderate growth failure}

Hwa V, Haeusler G, Pratt KL, Little BM, Frisch H, Koller D, Rosenfeld RG

Department of Pediatrics, Oregon Health and Science University Portland, Oreg., USA

hwav@ohsu.edu

J Clin Endocrinol Metab 2006;91:1826-1831

Background: IGF-1 circulates as part of a ternary complex with IGF-binding protein (IGFBP)-3 and acidlabile subunit (ALS) and may mediate the growth-promoting effects of GH. Another patient with inactivation of the ALS gene has been described [9], but due to the absence of family history and delayed puberty, the impact on growth was unproven. 
Methods: A patient in which serum IGF-1 and IGFBP-3 were abnormally low ( -5.8 and -7.2 SDS), but growth failure was modest ( -2.1 SDS at 15.5 years), was investigated.

Results: A novel homozygous missense mutation was identified in the ALS gene, which resulted in undetectable levels of serum ALS.

Conclusions: ALS is critical for maintaining normal serum concentrations of IGF-1 and IGFBP-3. ALS deficiency can be associated with moderate growth failure, and does not seem to affect the onset and progression of puberty.

IGF-1 circulates as ternary complex with IGFBP-3 and an ALS. This paper reports a patient with a novel homozygous missense mutation in the ALS gene resulting in undetectable ALS. Despite this, growth was minimally affected although the circulating concentrations of IGF-1 and IGFBP-3 were very low. This observation mirrors the phenotype of ALS-deficient mice [10] and confirms the phenotype of ALS deficiency in humans [9] and argues for a modest role for the ternary complex in the regulation of stature.

\title{
Reviews
}

\section{Endocrine regulation of human fetal growth: the role of the mother, placenta, and fetus}

Murphy VE, Smith R, Giles WB, Clifton VL

Mother and Baby Research Centre, Hunter Medical Research Institute, University of Newcastle, N.S.W., Australia

vicki.clifton@newcastle.edu.au

Endocr Rev 2006:27:141-169

\begin{abstract}
The environment in which the fetus develops is critical for its survival and long-term health. The regulation of normal human fetal growth involves many multidirectional interactions between the mother, placenta, and fetus. The mother supplies nutrients and oxygen to the fetus via the placenta and the fetus influences the provision of maternal nutrients via the placental production of hormones that regulate maternal metabolism. Endocrine regulation of fetal growth involves interactions between the mother, placenta, and fetus, and these effects may program long-term physiology.

This is an excellent overview of current concepts of the endocrinology of human fetal growth. It draws on both clinical observation, placental physiology and incorporates as appropriate animal data to support the contention that the endocrine system plays a greater role than hitherto thought.
\end{abstract}

\section{Food for thought}

Exon-3-deleted growth hormone receptor

\section{Growth hormone (GH) pharmacogenetics: influence of GH receptor exon-3 retention or deletion on first year growth response and final height in patients with severe GH deficiency}

Jorge AA, Marchisotti FG, Montenegro LR, Carvalho LR, Mendonca BB, Arnhold IJP

Hospital das Clinicas, Laboratorio de Hormonios, São Paulo, Brazil

alexj@usp.br

J Clin Endocrinol Metab 2006;91:1076-1080

Background: A polymorphism in the gene encoding the $\mathrm{GH}$ receptor $(G H R)$ that results in the exclusion of exon-3 (GHRd3) with the consequent removal of 22 amino acids is associated with better first- and second-year growth responses to recombinant human growth hormone (rhGH) in patients with idiopathic short stature or those who were born small-for-gestational [8]. The aim of this study was to 
study the effect of GHR-exon-3 genotype on the short- and long-term response to GH treatment in children with GHD.

Methods: Genotype and retrospective data collection was performed on 75 children with GHD. Patients were divided into two groups based on the genotype: full-length $(f l)$ and exon-3-deleted $(d 3$, homozygous and heterozygous) alleles. First-year growth velocity $(\mathrm{n}=58$, prepubertal) and adult height $(\mathrm{n}=44)$ after $7.5 \pm 3.0$ years of treatment were the main outcome measures.

Results: Patients carrying at least one GHRd3 allele had a significantly better growth velocity in the first year of GH treatment $(12.3 \pm 2.6$ vs. $10.6 \pm 2.3 \mathrm{~cm} /$ year; $\mathrm{p}<0.05)$ and achieved a taller adult height (final height SDS $-0.8 \pm 1.1$ vs. $-1.7 \pm 1.2 ; \mathrm{p}<0.05$ ) when compared with patients homozygous for GHRfl alleles.

Conclusion: In this study, patients with GHD who were homozygous for GHR exon-3-fl responded less well to rhGH treatment.

\section{The growth hormone (GH) response to GH treatment in children with isolated GH deficiency is independent of the presence of the exon-3-minus isoform of the GH receptor}

Blum WF, Machinis K, Shavrikova EP, Keller A, Stobbe H, Pfaeffle RW, Amselem S

Lilly Research Laboratories, Eli Lilly \& Co., Bad Homburg, Germany

Blum_Werner@Lilly.com

J Clin Endocrinol Metab 2006;91:4171-4174

Background: The aim of this study was to examine the impact of the GHR genotype on the phenotype and growth response in patients with isolated GH deficiency (IGHD) treated with GH.

Methods: A retrospective, multinational, multicenter observational study of 107 patients with IGHD. Genotypes ( $f l-G H R / f l-G H R, f l-G H R / d 3-G H R$, or $d 3-G H R / d 3-G H R$ ) were correlated with height SDS, height velocity, height velocity SDS at baseline and 1 year of GH treatment, and the changes in these parameters over 1 year of treatment.

Results: No statistically significant differences were observed between patients with the $d 3-G H R$ allele $(\mathrm{n}=48)$ and patients who were homozygous for the $f l-G H R$ allele $(\mathrm{n}=59)$. The mean serum IGF-1 concentration was lower in the $f l f l$ group, but this did not reach statistical significance.

Conclusion: The data suggest that the presence of the $d 3-G H R$ allele does not influence response to standard replacement doses of rhGH in a cohort of GHD children.

These two studies evaluating the effect of the presence of the $d 3-G H R$ allele on response to rhGH treatment in a cohort of patients with GHD appear to contradict each other. The reasons for this discrepancy remain open to speculation. Blum et al. suggest that the impact of the GHR genotype on $\mathrm{GH}$ responsiveness may be a dose-dependent phenomenon, and may be apparent only when supraphysiological doses of rhGH are used in SGA and Turner syndrome. Further studies will be required to clarify these issues.

References

1. Carta C, Pantaleoni F, Bocchinfuso G, Stella L, Vasta I, Sarkozy A, et al: Germline missense mutations affecting KRAS isoform B are associated with a severe Noonan syndrome phenotype. Am J Hum Genet 2006;79:129-135.

2. Roberts AE, Araki T, Swanson KD, Montgomery KT, Schiripo TA, Joshi VA, et al: Germline gain-of-function mutations in SOS1 cause Noonan syndrome. Nat Genet 2007;39:70-74.

3. Binder G, Wittekindt N, Ranke MB: Noonan syndrome: genetics and responsiveness to growth hormone therapy. Horm Res 2007;67(suppl 1):45-49.

4. Waxman DJ, O'Connor C: Growth hormone regulation of sex-dependent liver gene expression. Mol Endocrinol 2006;20:2613-2629.

5. Yakar S, Liu JL, Stannard B, Butler A, Accili D, Sauer B, et al: Normal growth and development in the absence of hepatic insulin-like growth factor-1. Proc Natl Acad Sci USA 1999;96:7324-7329.

6. Sjogren K, Liu JL, Blad K, Skrtic S, Vidal O, Wallenius V, et al: Liver-derived insulin-like growth factor 1 (IGF-1) is the principal source of IGF-1 in blood but is not required for postnatal body growth in mice. Proc Natl Acad Sci USA 1999;96:7088-7092.

7. Kofoed EM, Hwa V, Little B, Woods KA, Buckway CK, Tsubaki J, et al: Growth hormone insensitivity associated with a STAT5b mutation. N Engl J Med 2003;349:1139-1147.

8. Hwa V, Camacho-Hubner C, Little BM, David A, Metherell LA, El-Khatib N, et al: Growth hormone insensitivity and severe short stature in siblings: a novel mutation at the exon 13-intron 13 junction of the STAT5b gene. Horm Res 2007;68:218-224. 
9. Domene HM, Bengolea SV, Martinez AS, Ropelato MG, Pennisi P, Scaglia P, et al: Deficiency of the circulating insulinlike growth factor system associated with inactivation of the acid-labile subunit gene. N Engl J Med 2004;350:570-577.

10. Ueki I, Ooi GT, Tremblay ML, Hurst KR, Bach LA, Boisclair YR: Inactivation of the acid-labile subunit gene in mice results in mild retardation of postnatal growth despite profound disruptions in the circulating insulin-like growth factor system. Proc Natl Acad Sci USA 2000;97:6868-6873.

11. Gelb BD, Tartaglia M: Noonan syndrome and related disorders: dysregulated RAS-mitogen activated protein kinase signal transduction. Hum Mol Genet 2006;15(Spec No 2):R220-R226. 
